\title{
Conservative mitotic localization and functions of the nucleolar RPF2/Non3 protein in human and Drosophila cells
}

\author{
Svetlana V. Maltseva \\ Institute of Molecular and Cellular \\ Biology SB RAS, \\ Novosibirsk State University \\ Novosibirsk, Russian Federation \\ Evgenia S. Omelina \\ Institute of Molecular and Cellular \\ Biology SB RAS, \\ Novosibirsk, Russian Federation \\ Julia V. Popova \\ Institute of Molecular and Cellular \\ Biology SB RAS, \\ Novosibirsk, Russian Federation
}

\author{
Lybov A. Yarinich \\ Institute of Molecular and Cellular \\ Biology SB RAS, \\ Novosibirsk State University \\ Novosibirsk, Russian Federation \\ Alena V. Razuvaeva \\ Institute of Molecular and Cellular \\ Biology SB RAS, \\ Novosibirsk, Russian Federation \\ Anastasia A. Yushkova \\ Institute of Molecular and Cellular \\ Biology SB RAS, \\ Novosibirsk State University \\ Novosibirsk, Russian Federation \\ Gera A. Pavlova \\ Institute of Molecular and Cellular \\ Biology SB RAS, \\ Novosibirsk, Russian Federation
}

\author{
Anna A. Ogienko \\ Institute of Molecular and Cellular \\ Biology SB RAS, \\ Novosibirsk, Russian Federation
}

\author{
Alexey V. Pindyurin \\ Institute of Molecular and Cellular \\ Biology SB RAS, \\ Novosibirsk, Russian Federation \\ Evgenia N. Andreyeva \\ Institute of Molecular and Cellular \\ Biology SB RAS, \\ Novosibirsk, Russian Federation \\ andreeva@mcb.nsc.ru
}

\begin{abstract}
The nucleolus is a non-membrane nuclear organelle playing a crucial role in ribosome biogenesis in eukaryotes. Its morphology and function are associated with cell growth and proliferation, as well as with cell cycle regulation. Besides that, a set of nucleolar proteins are also known to be directly involved in mitotic cell division, taking a part in the spindle assembly. Among them is the human RRS1 (Ribosome biogenesis regulator 1 homolog) protein that function in complex with RPF2 (Ribosome production factor 2 homolog) in the ribosome biogenesis pathway. Here, we report the characterization of the mitotic localization and function of the human RPF2 and its Drosophila ortholog, the Non3 (Novel nucleolar protein 3 ) protein. We demonstrated that depletion of RPF 2 using siRNA specific to all its splicing variants leads to abnormal mitotic chromosome segregation in HeLa S3 cells. Similarly, the RNAi-mediated depletion of Non3 in Drosophila S2 cells also resulted in mitotic spindle defects including those in sister chromatid separation and segregation. Thereby, it will be possible to investigate mitotic functions of the RPF2 protein in Drosophila model system.
\end{abstract}

Keywords - nucleolus, RRS1, RPF2, Non3, moonlighting protein, mitosis, chromosome periphery, chromosome segregation defects

\section{Motivation and Aim}

The nucleolus is a non-membrane nuclear organelle playing a crucial role in ribosome biogenesis in eukaryotes. Its morphology and function are associated with cell growth and proliferation, as well as with cell cycle regulation. Besides that, a set of nucleolar proteins are also known to be directly involved in mitotic cell division, taking a part in the spindle assembly. Among them is the human RRS1 (Ribosome biogenesis regulator 1 homolog) protein that function in complex with RPF2 (Ribosome production factor 2 homolog) in the ribosome biogenesis pathway. RRS1 localizes at the periphery of the mitotic chromosomes and its depletion results in impaired chromosome alignment in metaphase and premature sister chromatid separation [1].
Currently, the information about localization of the RPF2 protein during mitosis is scarce [2] and nothing is known about its possible involvement in this process. However, the Drosophila ortholog of RPF2, the Non3 (Novel nucleolar protein 3) protein, was revealed to be somehow connected with the regulation of mitotic spindle length despite its exclusive localization in the nucleolus [3].

\section{Results}

We thoroughly studied the subcellular localization of all annotated isoforms of the RPF2 and Non3 proteins in human HeLa S3 and Drosophila S2 cells, respectively. For that, each protein was expressed as a GFP fusion and the cells were analyzed by confocal microscopy. We found that only the longest isoform (\#1) of RPF2 demonstrates specific localization at the chromosome periphery in metaphase. Both shorter isoforms (\#2 and \#X1) of RPF2, that lack the first 28 and 53 a.a. of the 190-a.a. long BRIX domain required for interaction with rRNAs, were found to form multiple aggregates dispersed between the metaphase chromosomes instead of covering the outer surface of chromosomes as the longer isoform does. Also, we were able to detect an enrichment of the single known isoform of Non3 around the metaphase plate. Next, we demonstrated that depletion of RPF2 using siRNA specific to all its splicing variants leads to abnormal mitotic chromosome segregation in HeLa S3 cells. Similarly, the RNAi-mediated depletion of Non3 in Drosophila S2 cells also resulted in mitotic spindle defects including those in sister chromatid separation and segregation. To analyze tissue-specificity of the phenomenon, we generated a number of Drosophila Non3 null and hypomorphic mutations. Immunostaining experiments performed on diploid Drosophila tissues suggested that centromere chromatin assembly is likely to be affected in Non3 mutants. 


\section{Conclusion}

The localization of the nucleolar RPF2 and Non3 proteins during mitosis is similar in human and Drosophila cells. In addition, mitotic defects observed upon depletion of these proteins are very similar suggesting the existence of functional conservation as well. Thus, Drosophila appears to be a relevant model system to study mitotic functions of the RPF2 protein.

\section{Acknowledgments}

This work was supported by the grant from the Russian Foundation for Basic Research (project \#18-34-00699).

\section{References}

[1] Gambe AE, Matsunaga S, Takata H, Ono-Maniwa R, Baba A, Uchiyama S, Fukui K. A nucleolar protein RRS1 contributes to chromosome congression. FEBS Lett. 2009, 583(12):19511956.

[2] Hirano Y, Ishii K, Kumeta M, Furukawa K, Takeyasu K, Horigome T. Proteomic and targeted analytical identification of BXDC1 and EBNA1BP2 as dynamic scaffold proteins in the nucleolus. Genes Cells. 2009, 14(2):155-166.

[3] Moutinho-Pereira S, Stuurman N, Afonso O, Hornsveld M, Aguiar P, Goshima G, Vale RD, Maiato H. Genes involved in centrosome-independent mitotic spindle assembly in Drosophila S2 cells. Proc Natl Acad Sci USA. 2013, 110(49):19808-19813. 